\title{
DEFECT GRAINS IN THE MELT-BACK REGION OF CMSX-4 SINGLE CRYSTAL SEEDS
}

\author{
N. Stanford ${ }^{1}$, A. Djakovic ${ }^{1}$, B. Shollock ${ }^{1}$, M. McLean ${ }^{1}$, N. D’Souza ${ }^{2}$, P. Jennings ${ }^{3}$. \\ ${ }^{1}$ Department of Materials, Imperial College London, Prince Consort Road, SW7 2AZ, London, United Kingdom. \\ ${ }^{2}$ Precision Casting Facility, PO Box 31, Rolls-Royce plc, Derby DE24 8BJ, United Kingdom. \\ ${ }^{3}$ Company Research and Development Foundry, PO Box 3, Rolls-Royce plc, Filton, Bristol BS34 7QE, United Kingdom.
}

Keywords: single crystal, seeds, stray grains, EBSD, orientation distribution, grain growth

\begin{abstract}
The nickel-based superalloy CMSX-4 is used commercially to cast single crystal components. During casting, a pre-fabricated seed is used to nucleate the desired orientation. During casting the top portion of the seed is partially melted and this mushy zone is the origin of defect stray grains. A number of seeds cast at RollsRoyce CRDF that exhibit extensive defect grain formation in the melt-back region have been analysed using optical and electron microscopy techniques. These defect grains were found to nucleate exclusively at the casting surface where the alloy contacts the mould. Nucleation of the defect grains is a transient behaviour, it occurs only during the preliminary stages of withdrawal. After this time, no further defect grain nucleation was observed. Due to growth propensity effects, non-axially oriented seeds exhibit more prolific defect grains towards one side of the casting.
\end{abstract}

\section{Introduction}

Single crystal turbine blades are state of the art in modern gas turbines for aircraft propulsion and the technology is being developed to produce the large castings required for power generation.[1] The benefits of single-crystal, over conventionallycast and directionally-solidified, components critically depends on being able avoid the introduction of casting defects, such as stray grains, freckles or deviations from the required crystal orientation.[2] Otherwise, either the property specifications must be relaxed or an economically unacceptable scrap-rate must be accepted. Two aspects of single-crystal superalloy development have increased the likelihood of solidification defects forming in industrial practice:

i. The refractory element additions, such as Rhenium, added to Second and Third generation SX superalloys to enhance the creep performance and alloy stability in service, have had a detrimental effect on their casting characteristics. This has led to difficulties in controlling the single crystal orientation and to an increased occurrence of stray grains and freckle chains.[3]

ii. The thermal conditions in producing large castings for industrial gas turbines are quite different from those experienced in the manufacture of turbine blades for aeroengines. In particular, the critical solidification parameters, temperature gradient and solidification rate are significantly reduced and the solidification front tends to be macroscopically curved, rather than planar. These factors combine to produce conditions where solidification defects are more likely to occur.[4]
For most SX castings the natural (001) solidification direction produces the preferred axial orientation for blades; this coincides with the direction of minimum Young's Modulus and leads to optimum thermal fatigue resistance. However, such natural growth does not determine the transverse orientation of the blade. There are also situations where axial orientations other than (001) are specified, for example to avoid resonance in operation. In these situations greater control of the crystal orientation is required.

Industrial casting of CMSX-4 turbine blades often involves the use of an oriented single crystal seed to nucleate the desired orientation for directional solidification. The pre-fabricated seed is placed in the mould, the entire assembly preheated, and then molten CMSX-4 is poured into the mould cavity. During the preheating step the top portion of the seed is melted, producing a liquid/solid region in the seed, referred to as the melt-back mush. During casting, this melt-back region can be the origin of stray grains that constitute defects in the final casting. Between the seed and the cast component, a "pig tail" orientation selector is used to eliminate these defective stray grains from the end product. If the defect grains at the seed/mush interface can be eliminated the casting procedure could be simplified by elimination the pig tail from the assembly. This would allow an efficient transfer of orientation from the high quality prefabricated seed directly into the cast part. A selection of five of these seeds showing extensive stray grain defects from this meltback region have been supplied by Rolls-Royce Company Research and Development Foundry (CRDF) for analysis. The aim of this work is to fully characterise these defect grains, and to attempt to determine their origin.

\section{Experimental Procedure}

All casting was carried out at the Rolls-Royce Company Research and Development Foundry in conditions that simulate industrial practice. The ceramic shell-mould was preheated and held at temperature for a 20 minute dwell prior to the start of the pour cycle. The liquid alloy was $\sim 1525^{\circ} \mathrm{C}$ when poured into the mould, and the assembly was withdrawn immediately after pouring at a rate of $3.8 \mathrm{~mm} \mathrm{~min}$. . In total, five seeds were supplied by the CRDF, and their characteristics are summarised in Table 1. Samples varied in their orientation and shape, but all had a maximum diameter of $12 \mathrm{~mm}$.

These castings showing defect grains forming at the seed/mush interface were examined using a range of microscopic techniques. For optical microscopy, samples were electro-etched in a solution of $10 \% \mathrm{HCl}$ in methanol for $2-3$ seconds at 5 volts. For electron microscopy samples were electro-polished in a solution of $45 \%$ 
butanol, $45 \%$ acetic acid and $10 \%$ perchloric acid at 25 volts for approximately 15 seconds. Electron micrographs were taken using backscattered imaging in a JEOL T200 scanning electron microscope (SEM), JEOL 840 SEM, or a LEO Gemini field emission gun SEM.

Since optical and electron microscopy techniques are affected by compositional differences inherent in the dendritic structures, the only technique able to reveal all stray grains within the microstructure was electron backscattered diffraction (EBSD). EBSD was carried out on electro-polished samples using two systems, a JEOL 840 SEM equipped with the HKL Channel 4.2 analysis package, and a LEO Gemini field emission gun SEM equipped with Oxford Instruments Crystal analysis package.

Table 1: Summary of the five seeds analysed. Orientation is the crystallographic direction of the seed that is parallel to the casting direction.

\begin{tabular}{|c|c|l|}
\hline Sample Ref. & Seed Orientation & \multicolumn{1}{|c|}{ Seed shape } \\
\hline RR01 & $<001>$ & Cylinder \\
\hline RR02 & $<015>$ & Cylinder (Figure 1a) \\
\hline RR03 & $<1214>$ & $\begin{array}{l}\text { Conical - seed tapers toward } \\
\text { casting direction }\end{array}$ \\
\hline RR04 & $<013>$ & $\begin{array}{l}\text { Almost cylindrical with one } \\
\text { flat side (Figure 1b). }\end{array}$ \\
\hline RR05 & $<128>$ & $\begin{array}{l}\text { Almost cylindrical with one } \\
\text { flat side (Figure 1b). }\end{array}$ \\
\hline
\end{tabular}

\section{Results}

\section{Macroscopic observation}

Samples were received with a macro-etch already applied and were photographed in this condition before sectioning. On all samples this macro-etch revealed stray grains that were easily seen with the naked eye, and an example is shown in Figure 1a.

\section{Optical and electron microscopy}

Two of the samples supplied (RR04 and RR05) were cylindrical with one flat face (illustrated in Figure 1b). This flat side was polished lightly and an example of the microstructure observed using optical microscopy is shown in Figure 1c. Long stray grains are clearly visible on the surface at this low magnification, and they extend many millimetres in the casting direction.

An example of the microstructure observed using electron microscopy is shown in Figure 2. This micrograph shows 3 distinct microstructural features; the solid seed, the melted-back portion of the seed, and the directionally solidified melt. The authors have reported the results of a detailed analysis of the the structure of this melt-back region aimed at discriminating between various proposed mechanisms of stray grain formation. [5] Electron microscopy showed that the microstructure of samples RR04 and RR05 on the flat faces were quite similar. Long stray grains are evident using backscattered imaging; however, not all of the defect grains are revealed, and their boundaries are not clearly defined. For this reason, these grains were further analysed using EBSD. An example of this orientation mapping of sample RR04 is shown in Figure 3. This is accompanied by an electron micrograph of the mapped region.

As can be seen from Figure 3b, there are a large number of grains formed on the surface of this sample. In the $5 \mathrm{~mm}$ square section analysed in Figure 3b there are 165 grains, and they have an average size of $240 \mu \mathrm{m}$. Many of the smaller grains close to the melt-back interface are equiaxed in shape, while those that continue to grow develop a columnar morphology.

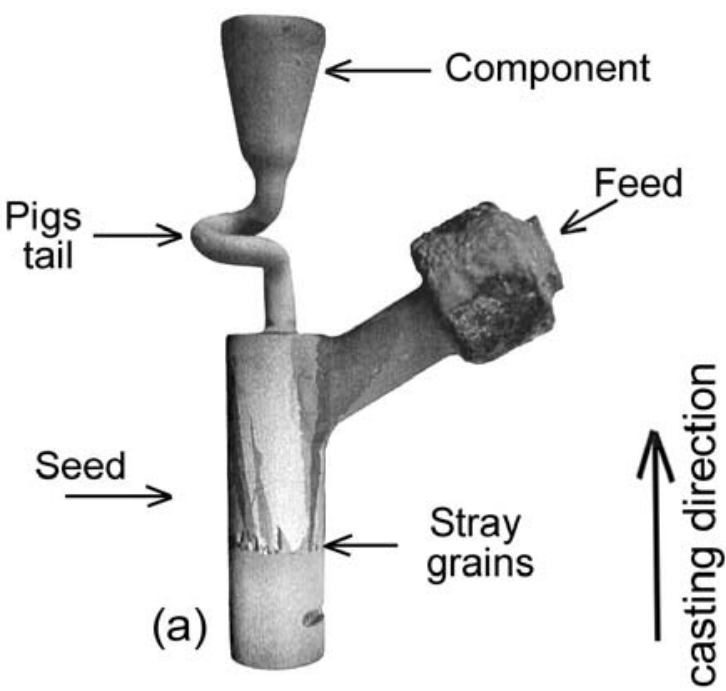

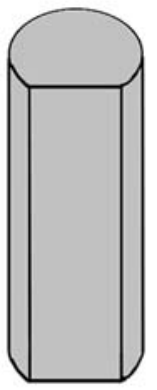

(b)

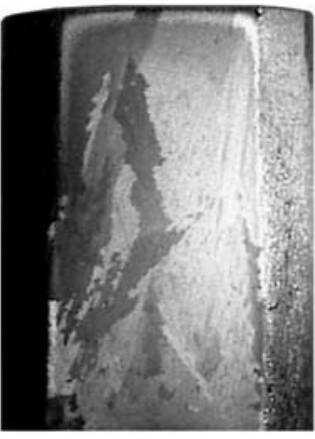

(c)
Figure 1: (a) Photograph of sample number RR02 showing stray grains. (b) Schematic illustration of the seed shape of samples RR04 and RR05. (c) Digital micrograph of flat face from sample RR04. Scale: All samples have a diameter of $12 \mathrm{~mm}$.

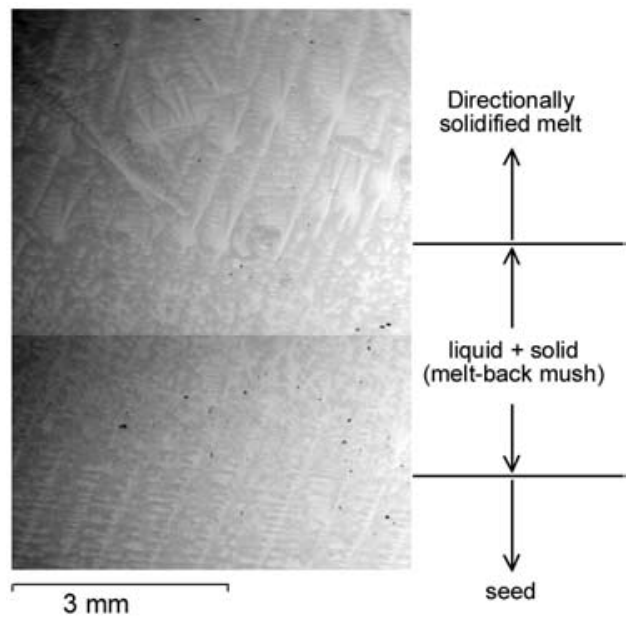

Figure 2: Electron micrograph of the melt-back region on the flat face of sample RR05. 


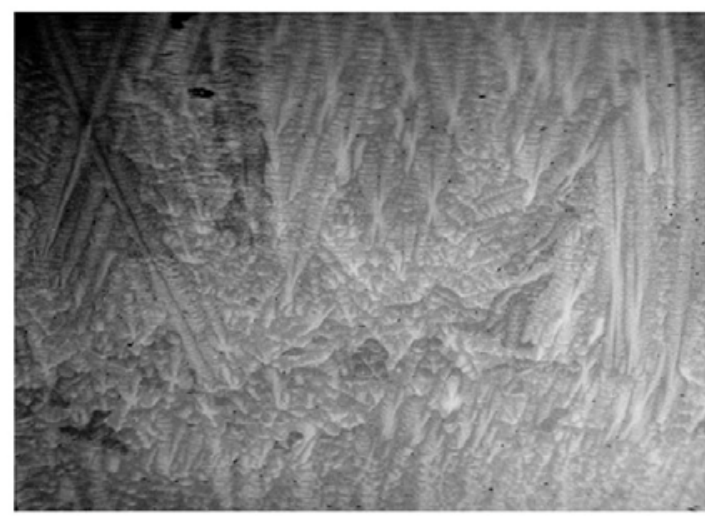

$3 \mathrm{~mm}$

(a)

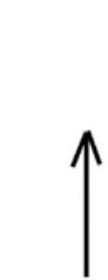

Casting direction

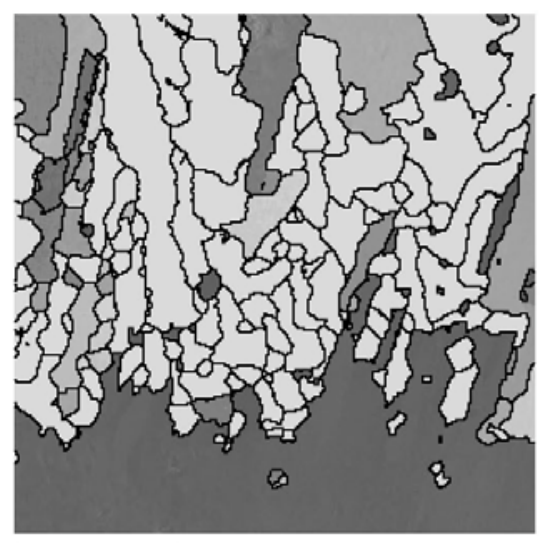

$1500 \mu \mathrm{m}$

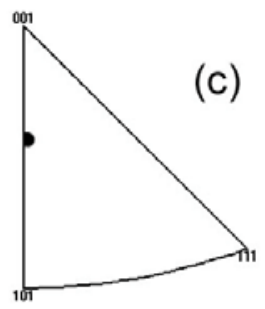

(d)

(a) Electron micrograph and (b) EBSD analysis of the flat face of sample RR04 at the interface between melt-backrefer to the casting direction.

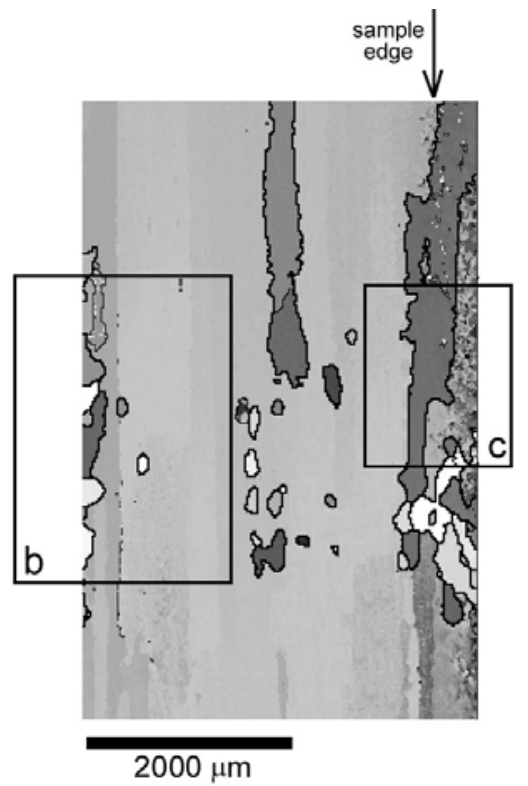

(a)

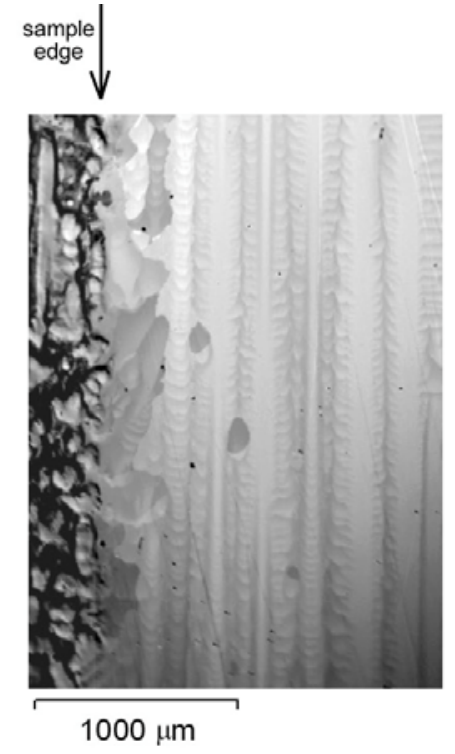

(b)

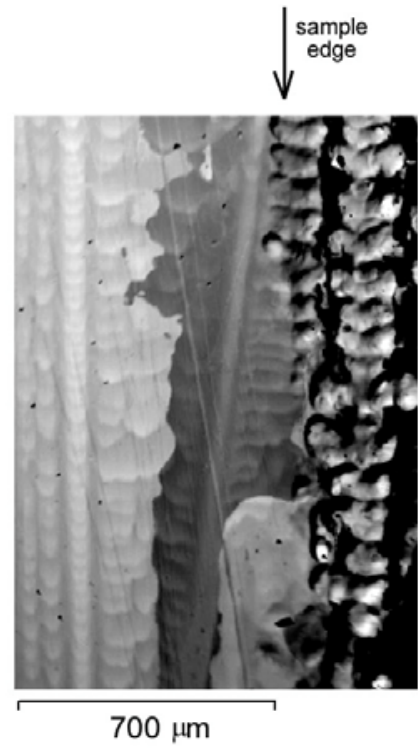

(c)

Figure 4: (a) EBSD micrograph of longitudinal section from shallow polishing of sample RR01 on the (100) plane. Intersection of the polished face and sample edge is arrowed. Higher magnification electron micrographs of the sections outlined in (a) are shown in (b) and (c). 
The average aspect ratio of the grains in Figure $3 b$ is 2.1 . The grains are random in orientation (Figure 3d), they do not have an orientation relationship to the parent seed or the casting direction. The onset of stray grain formation at the interface between meltback-mush and solidified melt appears quite uniform when viewed using backscattered imaging (Figure 3a). However, Figure $3 \mathrm{~b}$ shows that the nucleation of stray grains occurs over a distance of around $2000 \mu \mathrm{m}$ in the casting direction. In other words, new grains can be seen to nucleate well above the meltback mush.

Surface grains on sample RR01 were analysed by lightly polishing the cylindrical seed to produce a flat surface $3-5 \mathrm{~mm}$ wide on an area of interest. Polishing a shallow flat removes 100$300 \mu \mathrm{m}$ from the surface at the centre of the flat. An example of EBSD analysis on a shallow polished flat face from sample RR01 is shown in Figure 4.

Figure $4 \mathrm{a}$ shows the stray grains concentrated at the sample surface. Higher magnification electron micrographs of these surface grains are shown in Figs. 4b-c. Some small grains can be seen in the centre of the polished flat in Figure $4 \mathrm{a}$. These probably originated on the surface that has been polished away. Figure $4 \mathrm{~b}$ shows that the stray grains near the melt-back mush (within $5 \mathrm{~mm}$ ) are confined to the proximity of the surface of the casting; they neither nucleate at nor grow towards the centre of the casting. Longitudinal sectioning perpendicular to the casting surface was used to examine how this depth changed with solidification distance. An EBSD orientation map of sample RR04, perpendicular to the flat face from Figure 3, is shown in Figure 5. The stray grains that continue to grow during solidification tend to be retained close to the casting surface, and to persist over several millimetres in the casting direction.

To further investigate the effect of casting distance on the morphology and orientation of stray grains, sample RR02 was sectioned in the transverse direction in 5 places. Optical microscopy and EBSD were used to determine the size and shape of the defect grains that persisted over large solidification distances. The locations of the sections analysed are shown in Figure 6.

As can be seen, the stray grains are restricted to close to the surface of the casting for the first two sections (Figure 6c,d). However, a few grains at the perimeter of the casting continue to grow slowly expanding towards the centre, forming a crescentshaped outer edge. In the third section (Figure 6b), $14 \mathrm{~mm}$ from the melt-back/seed interface, three large grains can be clearly seen. Since small grains are not resolvable using optical techniques, selected sections were examined using EBSD. Examples are shown in Figures $7 \mathrm{a}$ and $7 \mathrm{~b}$. These microstructures have been used to quantify the effect of casting distance on the number of stray grains, and the maximum size of defect grains (Figure 8). As can be seen from the graph in Figure 8, the number of stray grains drops rapidly with casting distance. Figures $7 \mathrm{a}$ and $7 \mathrm{~b}$ show that the defect grains evident at melt-back have grown a significant distance from the casting surface toward the centre of the casting.
The defect grains increase rapidly in size with casting distance, and at the top of seed RR02 the largest grain had a $5 \mathrm{~mm}$ diameter in the transverse direction. EBSD on transverse sections also showed that at large casting distance only, the stray grains were more prolific towards one side of off-axis seeds (Figure 7b). This asymmetry is apparent on inspecting the macro-etched samples with the naked eye. Defect grains were found to occur on the side where the primary dendrites of the seed diverge from being parallel to the mould wall. For the large grains that have grown to long casting distances, this effect is very obvious (e.g. Figure 7b). However, sectioning close to the melt-back shows little bias inn stray grain nucleation; rather, the nucleated grains are distributed uniformly around the perimeter of the casting (Figure 7a). In sample RR01, the only $<001>$ seed orientation analysed, nucleation of defects occurred in clusters around the circumference. The grains that survived to large casting distances $\left(>6 \mathrm{~mm}\right.$ ) had misorientations of between $5^{\circ}$ and $25^{\circ}$ from the seed, and were positioned randomly around the circumference.

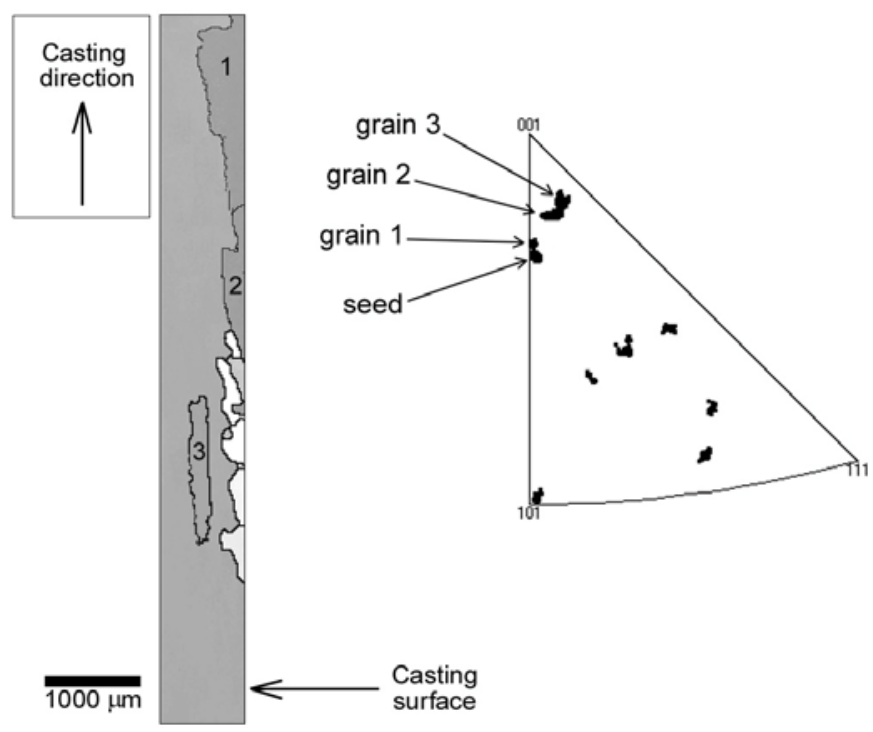

Figure 5: EBSD map of longitudinal section from sample RR04 perpendicular to the casting surface shown in Figure 3. Inverse pole figure refers to the casting direction. 


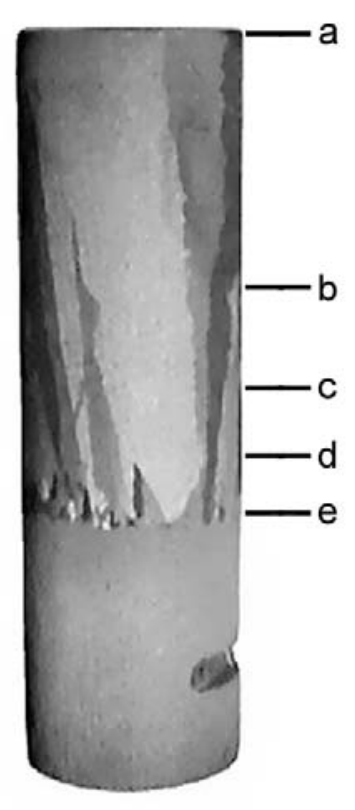

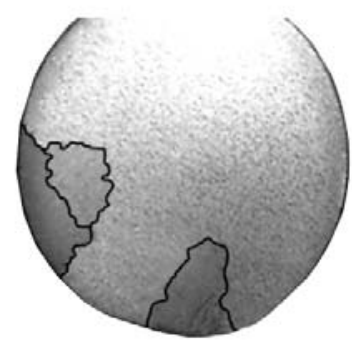

(a)

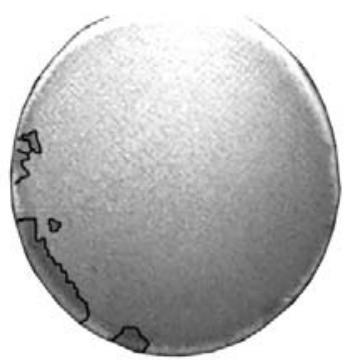

(c)

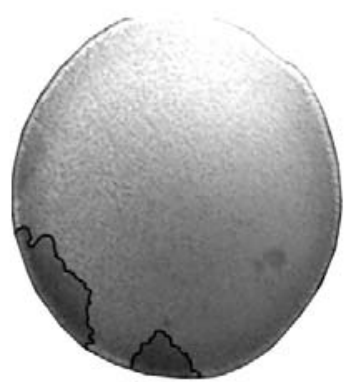

(b)

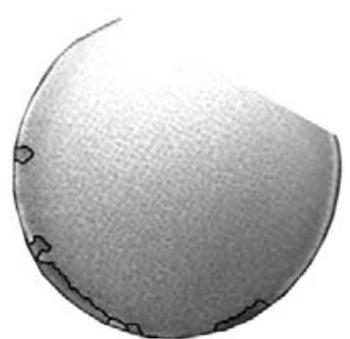

(d)

Figure 6: Optical micrographs of transverse sections of sample RR02. The figure on the left shows the approximate position of each section. The distances from point $\boldsymbol{e}$, the location of the melt-back/seed interface, to each section are: (a) $29.5 \mathrm{~mm}$ (b) $14 \mathrm{~mm}$ (c) $7 \mathrm{~mm}$ (d) $2.5 \mathrm{~mm}$. The sample diameter is $12 \mathrm{~mm}$.

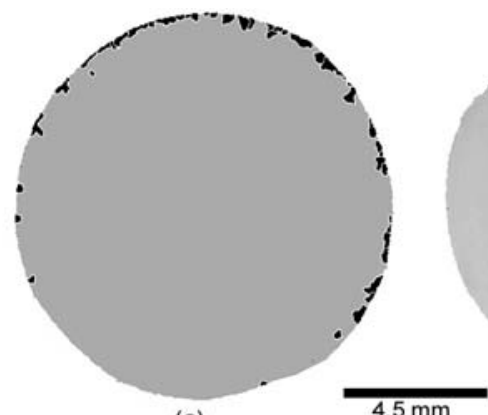

(a)

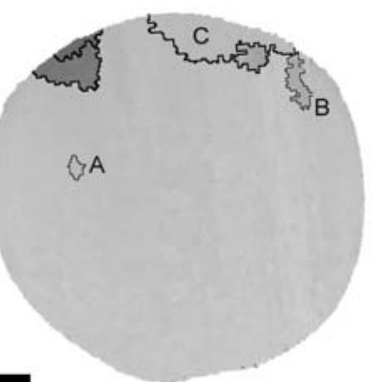

(b)

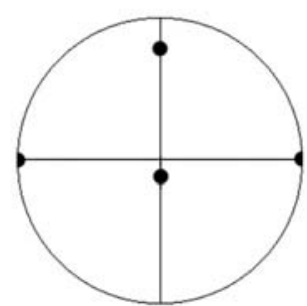

(c)

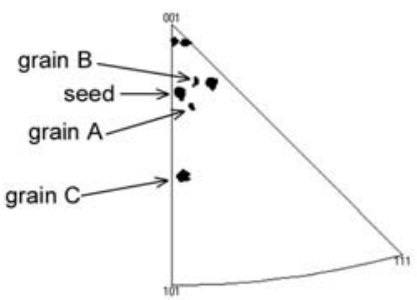

(d)

Figure 7: (a) EBSD analysis of transverse section of sample RR02 from section $\boldsymbol{e}$ in Figure 6. Grain boundaries have been outlined by hand for clarity. (b) EBSD analysis of RR02 section b. Grain boundaries larger than $2.5^{\circ}$ in misorientation have been outlined in fine black lines, boundaries greater than $10^{\circ}$ in misorientation have been outlined in bold black lines. The orientation of the seed is shown in the (100) pole figure in (c). The stray grain orientations from (b) are shown in the inverse pole figure of (d), referring to the casting direction. 


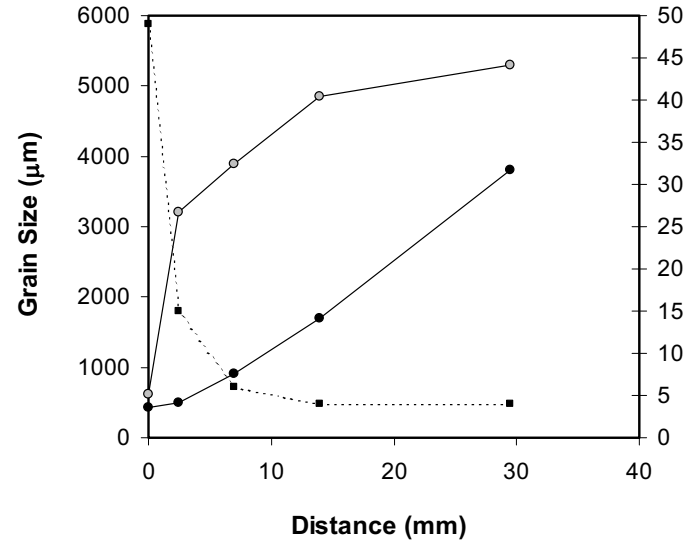

Grain length $\rightarrow-$ Grain width $\cdots \cdots$ Number of grains

Figure 8: Effect of casting distance on the morphology and number of defect stray grains. Grain length refers to the length of the largest grain around the sample circumference. Grain width refers to the largest distance the defect grains grow from the sample edge toward the sample centre. Only grains surrounded by high angle boundaries have been included in the analysis.

\section{Discussion}

\section{Orientation and growth potential}

Figure $3 \mathrm{~b}$ has been shaded so that the grains close in orientation to that of the seed are dark grey, and those grains far in orientation from that of the seed are light grey. The darker grains are in general columnar in shape, and are aligned parallel to the primary arms of the seed (towards the top right hand side of the page). The clusters of small equiaxed grains close to the meltback are highly misoriented from the seed.

The longitudinal section of Figure 5 shows the largest grain (grain 1) that has survived to a $5 \mathrm{~mm}$ casting distance has a small misorientation of $\sim 4^{\circ}$ from the seed. The other two large grains in Figure 5 (labelled grain 2 and 3) that are 1500 and $1600 \mu \mathrm{m}$ long respectively are also close in orientation to the seed. Figure 5 also shows that these two large grains are closer to $<001>$ than is the seed; they have a natural growth direction almost parallel to the casting direction. The cluster of nine smaller grains close to the melt-back, coloured light grey in Figure 5, have an average size of $260 \mu \mathrm{m}$. These small equiaxed grains have orientations far from that of the seed, and all have orientations remote from $<001>$.

EBSD on transverse sections at large casting distances $(29 \mathrm{~mm})$ revealed two types of defect grains. Firstly, there are grains that have grown into the centre of the casting and appear to be no longer connected to the casting surface (grains A and B in Figure $7 b$ ). These grains are surrounded by low angle boundaries, $\sim 3.5^{\circ}$ in both grains A and B. Secondly, there are defect grains that have remained at the casting surface, and these have boundary misorientations of between 12 and $28^{\circ}$ from the seed (e.g. grain $\mathrm{C}$ in Figure 7b).

The other orientation effect of interest is the bias of stray grains to one side of non-axial seeds. The transverse section in Figure 7 a shows that nucleation of stray grains occurs fairly uniformly around the circumference of the seed, whilst Figure $7 \mathrm{~b}$ shows that after some distance the defect grains only survive on one side of the seed. Specifically, the side of the seed where the primary dendrites of the seed diverge from being parallel to the mould wall is where these grains develop; where the dendrites converge on the mould wall the stray grains are over-grown by the seed crystal. The classical model of competitive growth between neighbouring dendrites proposed by Walton and Chalmers [6] has been shown to be to be inappropriate to account for grain competition in chemically complex alloys such as CMSX-4 [7]. The behaviour of competing dendrites is different depending on whether the primary dendrites of the two grains are converging towards one another or diverging. Where grains converge, solute rejection by both dendrite tips inhibits the growth of secondary arms producing a boundary parallel to the leading dendrite. The boundary between grains is not vertical, but angled dependent on the orientation of each dendrite. The sharper the angle, the faster one grain will out grow the other by intersection with the mould wall. Where grains diverge, secondary and tertiary arms are able to grow into the space between the adjacent grains [7-9]. Referring back to the non- $<001>$ seeds in the current study, on the side where the seed dendrites converge onto the mould wall, defect stray grains are quickly out grown by the seed dendrites. Where the seed primaries and the mould diverge, the stray grains are able to grow secondary and tertiary arms to fill the space where the seed dendrites grow away from the mould wall.

If the possibility of a concave solidification front is considered (eg. Figure 9d), then defect grains on the perimeter of the casting could be able to grow ahead of the central seed orientation, enhancing their ability to survive over-growth.

\section{Nucleation mechanisms}

Macroscopic and microscopic investigation has shown that after melt-back, surface grain defects nucleate only over a distance of $\sim 2 \mathrm{~mm}$ in the casting direction; after this distance no further nucleation is observed. At a withdrawal rate of $3.8 \mathrm{~mm} \mathrm{~min}^{-1}$, nucleation thus occurs over a time interval of $\approx 30$ seconds. This suggests that the formation of these defects is a transient behaviour that only occurs during the initial stages of withdrawal. In addition to these defects occurring in a small $2 \mathrm{~mm}$ vertical range, EBSD has shown that horizontally these grains are restricted to within $\sim 400 \mu \mathrm{m}$ of the cast surface at the point of nucleation. They are not observed to nucleate within the centre of the casting (e.g. Figure 7a).

Three mechanisms have been proposed for the nucleation of such stray grains:

i. Pinched-off secondary dendrites in the semi-solid region of the melted-back seed are thought to be transported by convective currents ahead of the dendrite tips to nucleate defect grains in the liquid that has been subject to solutal undercooling $[10,11]$. In a previous study we have analysed the misoriented regions in the melt-back zone.[5] Although pinched-off dendrites are observed, there are too few to account for the high density of surface stray grain nucleation that is observed. Also, they are uniformly distributed in the melt-back region and they are not observed ahead of the semi-solid region. Indeed, since the dendrites are significantly more dense than the inter-dendritic liquid, these fragment would be expected to sink rather than rise through the melt.

ii. Heterogeneous nucleation of grains can occur in front of the dendrite tips where there is solutal undercooling. High 
levels of nitrogen in some alloys allow precipitation of TiN particles ahead of the solidification front. This can lead to heterogeneous nucleation of stray grains on these particles $[12,13]$. The alloys used in the present experiments have low nitrogen contents and there is no evidence of nitride formation. Also, this mechanism would nucleate grains through out the casting rather than at the perimeter.

iii. Grains could form by heterogeneous nucleation on a lowtemperature seed or at the mould surface when introducing the alloy charge on top of the seed within the mould cavity. Pouring molten alloy into a colder seed/mould assembly may allow rapid nucleation of chill grains. Some of these grains may survive the equilibration time without melting or being overgrown in the early stages of directional solidification.

It is highly unlikely that these stray-grain defects can originate either from broken-off dendrite arm fragments or by nucleating at nitrides in the melt. Both mechanisms would produce solid fragments throughout the liquid; they would not form exclusively at the surface of the casting. Furthermore, neither mechanism would be transient; if either mechanism could produce defect grains at the beginning of solidification then they could also nucleate stray grains throughout casting, and this was not observed in the current study.

The most likely mechanism for the formation of stray grains is due to chilling of the liquid CMSX-4 when being poured into the mould. The seed, resting on a water-cooled chill is solid when the molten alloy is poured into the mould cavity. If the colder seed quenches the molten alloy as it enters the mould, stray grains could be formed across the seed diameter; however, stray grains are only observed at the surface of the casting. For this reason the seed chilling effect is not considered to be the cause of defect grains either.

Clearly, the defects are caused by conditions that are unique to the surface of the casting. More than likely, these grains are associated with radial heat loss during withdrawal of the seed from the furnace, producing a thermal gradient where the casting surface is colder than the centre (the mould is colder than the melt). If the temperature of the outer edges drops below the solidus temperature, chill grains could nucleate at the mould wall. If the isotherm were to change rapidly (such as during the initial stages of withdrawal) then an undercooled region of sufficient volume could allow heterogeneous nucleation of grains on the mould wall (shown schematically in Figures 9a-c). After a small amount of solidification, density inversion due to solute rejection into the liquid may allow solute enriched liquid to rise up the sides of the mould wall. This will slow the growth in these regions and allow the solidification front to equilibrate with the isotherm across the diameter of the seed [i.e. the system reaches steady state (Figure 9d)]. Essentially, these defective grains are thought to be caused by sudden radial heat loss.

\section{Stability of stray grains}

In view of the profuse nucleation stray grains on introducing the liquid metal charge into the seed assembly, it is unlikely that they could be eliminated without radical modification of industrial practice. The very obvious grains that are exposed by macro etching (Figure 1) show the very few of the nucleated grains that continue to grow and penetrate towards the centre of the casting. It is these grains that have the potential to pass through the grain selection mechanism into the component. Consequently, the problem is associated with the growth rather than nucleation of stray grains.

Clearly, most of the grains nucleated at the seed/melt interface have a transitory life and are quickly over-grown by the seed crystal. There are two factors that determine the few crystals that continue to grow at the expense of the seed crystal. Firstly, if the orientation of the stray grain parallel to the macroscopic solidification direction is closer to the fast growing (001) direction than is the seed crystal, then the seed will dominate. Secondly, if the solid/liquid interface is significantly concave upwards, an apparently unfavourable orientation can grow normal to the local solidification front.

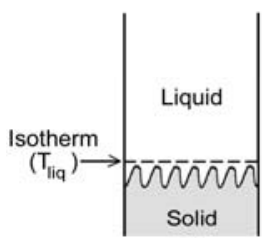

(a)

Equilibration

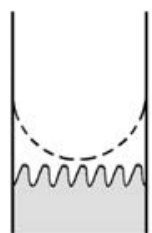

(b)

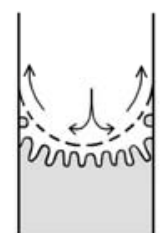

(c)
Preliminary solidification

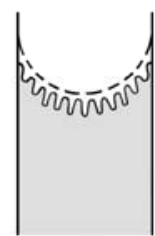

(d) state

Figure 9: Schematic illustration of surface defect grain formation during preliminary solidification of single crystal seeded CMSX-4.

\section{Industrial Relevance}

The present study relates to stray grains that nucleate due to local undercooling near the mould wall when introducing a liquid metal charge to a partially melted seed crystal. This is a highly localised event that can lead to the growth of a few unwanted preferentially oriented grains to the grain selector and then to the component. The present study provides guidance on how the heat flow may be managed to minimise this effect.

The features observed in this study are not related to the formation of slivers, which can be widespread over the length of the single crystal casting being associated with local dendrite growth normal to a highly curved macroscopic solid-liquid interface.

\section{Conclusions}

Defect stray grain formation during seeded casting is a surface based effect. Stray grains are not observed in the centre of castings. Nucleation of defect grains is transient, it occurs over a distance of $\sim 2 \mathrm{~mm}$ at the very beginning of withdrawal. Once nucleated, a few stray grains grow laterally to become crescent shaped, and with continued withdrawal these grains grow toward the centre of the casting. Defect grains are random in orientation when nucleated, but those that survive to large casting distances are generally aligned closer to $<001>$ than is the seed.

In seeds where $<001>$ is not parallel to the casting direction, defect grains are more prolific on the side of the seed where the primary dendrites diverge from the casting wall. This is a result of higher growth propensity, not necessarily due to a nucleation bias on one side. The nucleation event is not drastically affected by seed orientation.

It is proposed that surface defect grains form by heterogeneous nucleation of chill grains on the mould wall during preliminary solidification as a result of undercooled liquid ahead of the dendrite tips. 


\section{References}

1. P. Caron, and T. Khan, "Development of a New Nickel Based Single Crystal Turbine Blade Alloy for Very High Temperatures,"Advanced Materials and Processes, Vol. 1, ed. H.E. Exner and V.Schumacher (Oberursel, Germany: DGM InformationsgesellschaftmbH, 1990), 333-338.

2. S. Tin, T.M. Pollock and W.T. King, "Carbon Additions and Grain Defect Formation in High Refractory Nickel-Base Single Crystal Superalloys, pp 201-210, Proc. of $9^{\text {th }}$ International Symposium on Superalloys, Seven Springs, PA, 2000, (ed. T.Pollock et al.).

3. T.M. Pollock and, W.H. Murphy, "The Breakdown of Single-Crystal Solidification in High Refractory NickelBase Alloys", Metall. Mater. Trans. A, 27A (1996) 10811094

4. A.Lohmtillert, W. Esser, J. Grossmann, M. Hordler', J. Preuhs and R. F. Singer, "Improved Quality and Economics of Investment Casting By Liquid Metal Cooling -the Selection of Cooling Media", pp 181-188, Proc. of $9^{\text {th }}$ International Symposium on Superalloys, Seven Springs, PA, 2000, (ed. T.Pollock et al.).

5. N.Stanford, A.Djakovic, B.A.Shollock, M.McLean, N.D'Souza and P.Jennings, "Seeding of single crystal superalloys - role of seed melt-back on casting defects", Scripta Mater. 50 (1): 159-163, 2004

6. D. Walton and B. Chalmers, "The origin of the preferred orientation in the columnar zone of ingots" Trans. AIME., 215 (1959) p. 447-457.

7. M. Ardakani, N. D'Souza, A. Wagner, B. Shollock, M. McLean, "Competitive grain growth and texture evolution during directional solidification of superalloys" (paper presented at the $9^{\text {th }}$ International Symposium on Superalloys, Seven Springs, PA, 2000) p. 219-228.

8. N. D'Souza, M. Ardakani, A. Wagner, B. Shollock, M. McLean, "Morphological aspects of competitive grain growth during directional solidification of a nickel-base superalloy, CMSX-4", Journal of Materials Science, 37 (2002) p. 481-487.

9. A. Wagner, N. D'Souza, B. Shollock, and M. McLean, "Competitive grain growth and solute partitioning in nickelbase superalloys" (paper presented at the 2001 International Symposium on Liquid Metal Processing and Casting, Santa Fe, USA, 2001) p. 301-313.

10. K.A. Jackson, J.D. Hunt, D.R. Uhlmann and D.P. Seward, "On the origin of the equiaxed zone in casting", Trans. AIME., 236 (1966) p. 149-157.

11. J.P. Gu, C. Beckermann and A.F Giamei, "Motion and remelting of dendrite fragments during directional solidification of a nickel-base superalloy", Metallurgical and Materials Transactions A, 28A (1997) p. 1533-1542.

12. T. Wang, E. Lae, and A. Mitchell, "Nitride particles and grain nucleation in superalloy single crystal castings" (paper presented at the 2001 International Symposium on Liquid
Metal Processing and Casting, Santa Fe, USA, 2001) p. 280287.

13. X. Huang, Y. Zhang, Y. Liu, and Z. Hu, "Effect of small amount of nitrogen on carbide characteristics in unidirectional Ni-base superalloy", Metallurgical and Materials Transactions A, 28 A (10) (1997) p. 2143-2147.

\section{Acknowledgements}

The work described in this paper was carried out as part of EPSRC Grant GR/M720074/01. The authors would also like to thank Dr. Mahmoud Ardakani for his electron microscopy expertise and the CRDF at Rolls-Royce Bristol for the provision of materials. 